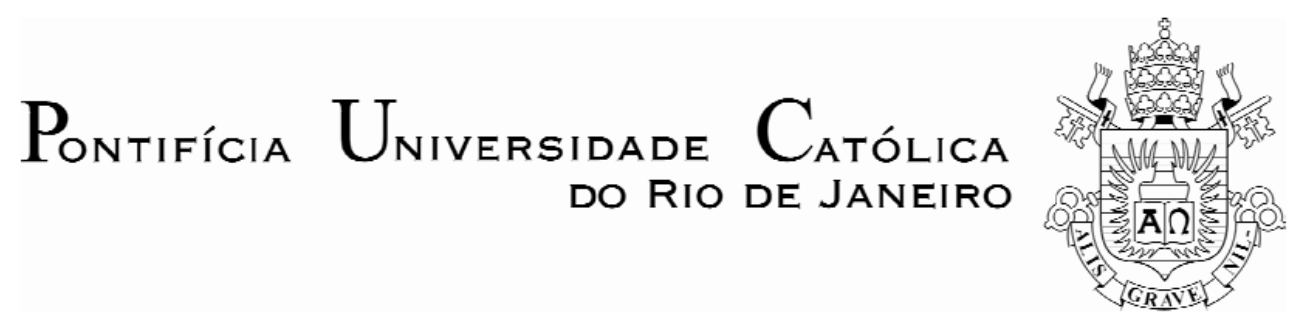

Eduardo Ferraz de Lima Vieira

\begin{abstract}
Avaliação de Projetos de Investimento em Plantas XTL utilizando a Teoria de Opções Reais
\end{abstract}

Dissertação de Mestrado

Dissertação apresentada como requisito parcial para obtenção do título de Mestre pelo Programa de PósGraduação em Engenharia Industrial da PUC-Rio.

Orientador: Prof. José Paulo Teixeira

Rio de Janeiro Março de 2007. 
Pontifícia Universidade $_{\text {Do Rio de Janeiro }}$

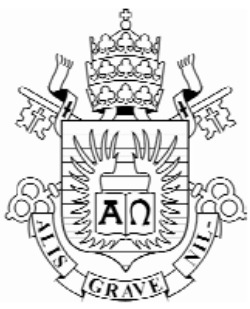

Eduardo Ferraz de Lima Vieira

\section{Avaliação de Projetos de Investimento em Plantas XTL utilizando a Teoria de Opções Reais}

Dissertação apresentada como requisito parcial para obtenção do título de Mestre pelo Programa de PósGraduação em Engenharia Industrial da PUC-Rio. Aprovada pela Comissão Examinadora abaixo assinada.

Prof. José Paulo Teixeira

Orientador

Departamento de Engenharia Industrial-PUC-Rio

Prof. Marco Antônio Guimarães Dias

Departamento de Engenharia Industrial-PUC-Rio

Prof. Carlos Patrício Samanez

Departamento de Engenharia Industrial-PUC-Rio

Prof. José Eugenio Leal Coordenador Setorial do Centro

Técnico Científico - PUC-Rio

Rio de Janeiro, 06 de março de 2007 
Todos os direitos reservados. É proibida a reprodução total ou parcial do trabalho sem autorização da universidade, do autor e do orientador.

\section{Eduardo Ferraz de Lima Vieira}

Formado em Engenharia Elétrica/Telecomunicações pela Pontifícia Universidade Católica do Rio de Janeiro. Participou do projeto XTL - Cenpes/Petrobras de análise de investimentos de uma planta GTL através da teoria de opções reais.

Ficha Catalográfica

Vieira, Eduardo Ferraz de Lima

Avaliação de projetos de investimento em plantas XTL utilizando a teoria de opções reais. Estudo de caso do projeto XTL - Cenpes/Petrobras / Eduardo Ferraz de Lima Vieira; orientador: José Paulo Teixeira. - 2007.

78 f.: il. ; $29,7 \mathrm{~cm}$

Dissertação (Mestrado em Engenharia Industrial)Pontifícia Universidade Católica do Rio de Janeiro, Rio de Janeiro, 2007.

Inclui referências bibliográficas

1. Engenharia industrial - Teses. 2. Análise de investimentos. 3. Teoria das opções reais. 4. Processo estocástico. 5. Movimento de reversão à media com saltos. 6. Viabilidade econômica. I. Teixeira, José Paulo. II. Pontifícia Universidade Católica do Rio de Janeiro. Departamento de Engenharia Industrial. III. Título. 


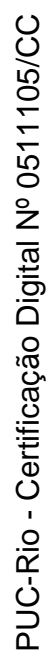

À minha família e futura esposa. 


\section{Agradecimentos}

Em especial ao professor José Paulo Teixeira, pelo total apoio, sacrifício e orientação no trabalho que foi realizado.

Ao professor Marco Antônio Dias, pela excelência no ensino da metodologia de opções reais e ao professor Carlos Patrício Samanez, pelas opiniões e sugestões de análise.

Ao Centro de Pesquisas e Desenvolvimento Leopoldo A. Miguez de Mello CENPES, pela iniciativa e suporte desta pesquisa e em especial a todos os colaboradores responsáveis pelo desenvolvimento do projeto $\mathrm{XTL}$, entre eles: Luis Alberto Melchíades, Alessandro Oliveira, Reynaldo Taylor, Luís Eduardo Peron, Sirlei Souza e Henrique S. Cerqueira. Gostaria de agradecer também ao colaborador da área de E\&P/Petrobras Edison Tito pelo auxílio na programação da plataforma computacional utilizada.

À Coordenação de Aperfeiçoamento de Pessoal de Nível Superior - CAPES, pelo apoio financeiro.

Por fim, à Pontifícia Universidade Católica por todo conhecimento transmitido ao longo de minha graduação em engenharia elétrica e pela excelência de conhecimento no programa de pós-graduação do departamento de engenharia industrial. 


\section{Resumo}

Ferraz de Lima Vieira, Eduardo; Teixeira, José Paulo. Avaliação de projetos de investimento em plantas XTL utilizando a teoria de opções reais. Rio de Janeiro, 2007. 78p. Dissertação de Mestrado - Departamento de Engenharia Industrial, Pontifícia Universidade Católica do Rio de Janeiro.

O objetivo da presente dissertação é capturar o valor da flexibilidade que uma planta XTL oferece na entrada do sistema produtivo, onde podem ser utilizados diversos tipos de insumos como matéria-prima. A saída do sistema produtivo também permite que diferentes produtos sejam produzidos. Desta forma, o autor considera que a metodologia das opções reais é a mais indicada para se avaliar tais flexibilidades, sendo o objeto principal deste estudo a análise da opção de conversão (Input/Output Switch option) através da utilização do processo estocástico de reversão à média com saltos. Os resultados desta dissertação podem auxiliar a tomada de decisão dos gestores da área de petróleo e energia, onde outros projetos já foram avaliados através dessa mesma metodologia, no entanto, não existe qualquer interesse em comprovar a eficiência da teoria das opções reais frente às metodologias de avaliação financeira usuais.

\section{Palavras-chave}

Análise de investimentos; teoria das opções reais; processo estocástico; movimento de reversão à media com saltos; viabilidade econômica. 


\section{Abstract}

Ferraz de Lima Vieira, Eduardo; Teixeira, José Paulo. Valuation of XTL Plants Project Investment based on Real Options Theory. Rio de Janeiro, 2007. 78p. MSc Dissertation - Departamento de Engenharia Industrial, Pontifícia Universidade Católica do Rio de Janeiro.

The objective of this dissertation is to value the operational flexibility that a GTL plant can offer at the entrance of the productive process where multiples inputs can be used as row material. Different products also can be produced. The real options theory is considered by the author as the most indicated financial methodology to value theses flexibilities and the main goal of the present study is to value the Input/Output switch option and the mean reversion with jumps stochastic process. The aim of this study is also to support manager's investment decision from oil and energy field, where others projects has been already valuated with real options theory so the proof of the theory efficiency against traditional valuation methodologies is not object from this study.

\section{Keywords}

Investment analysis; real option theory; mean reversion with jumps stochastic process; economic viability. 


\section{Sumário}

1 Introdução

20 setor Gas-to-Liquids (GTL)

2.1. Histórico

2.2. A capital mundial do GTL

2.3. Diesel GTL: O combustível limpo

2.4. O processo GTL + Gaseificador

2.4.1. Geração do Gás de síntese 22

2.4.2. Processo de Fischer-Tropsch 23

$\begin{array}{ll}\text { 2.4.3. Hidroprocessamento } & 24\end{array}$

3 Análise por Opções Reais 26

3.1. Exemplos de opções reais 27

3.2. A opção de conversão (Switch Use Option) 30

3.3. Simulação de Monte Carlo 32

4 A modelagem estocástica $\quad 34$

4.1. Definição 35

4.2. Principais processos estocásticos 37

4.2.1. Movimento Aritmético Browniano 38

4.2.2. Movimento Geométrico Browniano 39

4.2.3. Movimento de Reversão à Média 40

4.3. Lema de Itô 42

4.4. Movimento de Reversão à Média com Saltos 44

4.4.1. O modelo 45

5 Estudo de caso: Refinaria XTL 48

5.1. Precificação das opções de conversão 48

5.2. Premissas de simulação 50

5.2.1. Características técnicas dos inputs 50 
5.2.2. Capacidade nominal de produção

5.2.3. Capex $\left(\mathrm{I}_{0}\right)$

5.2.4. Custo dos produtos vendidos - CPV 52

5.2.5. Receitas operacionais 53

5.2.6. Impostos e Taxas $\quad 55$

5.2.7. Séries de Preços $\quad 55$

5.2.8. Aspectos financeiros 63

5.2.9. Outras premissas 64

6 Resultados e conclusões 65

6.1. Apresentação dos resultados 65

6.2. Conclusões 69

6.3. Sugestões para futuros trabalhos 70

7 Referências bibliográficas $\quad 71$

8 Apêndices e anexos $\quad 73$ 


\section{Lista de figuras}

Figura 1 - Processo Gas-to-Liquids $\quad 25$

Figura 2 - Flexibilidades em um projeto de investimento 27

Figura 3 - Combinação ótima de insumos e produtos do $\begin{array}{ll}\text { processo XTL } & 31\end{array}$

Figura 4 - Representação gráfica de simulação de Monte Carlo. 32

Figura 5 - Movimento Aritmético Browniano (MAB) 39

Figura 6 - Movimento Geométrico Browniano (MGB) 40

Figura 7 - Movimento de Reversão à Média (MRM) 41

Figura 8 - Preços nominais do óleo Brent e similares - período mensal (1970 a 2003) 44

Figura 9 - Distribuição de probabilidade dos saltos aleatórios 46

Figura 10 - Curvas de produção de líquidos sintéticos (ASF) 54

Figura 11 - Evolução histórica dos preços do gás natural -

evolução mensal 56

Figura 12 - Evolução histórica dos preços do óleo pesado -

evolução diária $\quad 56$

Figura 13 - Evolução histórica dos preços da Nafta - evolução mensal 57

Figura 14 - Evolução histórica dos preços do Diesel - evolução mensal 57

Figura 15 - Evolução histórica dos preços da Parafina e Lubrificantes evolução mensal 58

Figura 16 - Projeção real e neutra ao risco dos preços do gás natural projeção trimestral 60

Figura 17 - Projeção real e neutra ao risco dos preços do óleo pesado -

projeção trimestral

Figura 18 - Projeção real e neutra ao risco dos preços da nafta -

projeção trimestral

Figura 19 - Projeção real e neutra ao risco dos preços do diesel projeção trimestral

Figura 20 - Projeção real e neutra ao risco dos preços da parafina projeção trimestral

Figura 21 - Projeção real e neutra ao risco dos preços do lubrificante projeção trimestral

Figura 22 - Valor da opção em função do fator de correlação 66

Figura 23 - Sensibilidade do Valor presente Líquido 76

Figura 24 - Distribuições de probabilidade dos resultados encontrados 77 


\section{Lista de tabelas}

Tabela 1 - Principais plantas GTL no mundo 17

Tabela 2 - Principais acordos para desenvolvimento de plantas GTL 18

Tabela 3 - Lista dos índices de enxofre permitido nos combustíveis $\quad 20$

Tabela 4 - Propriedades do diesel GTL 21

Tabela 5 - Relação dos tipos de plantas industriais analisadas $\quad 49$

Tabela 6 - Rendimentos por tipo de input 50

Tabela 7 - Investimento por barril e capacidade de planta 51

Tabela 8 - Alocação de recursos por fase de projeto 51

Tabela 9 - Valor total de Investimentos para uma planta de $35.000 \mathrm{bbl} / \mathrm{dia}$

Tabela 10 - Quantidades diárias de matéria-prima 53

Tabela 11 - Perfil de produção 54

Tabela 12 - Parâmetros do processo estocástico de reversão

à média com saltos $\quad 59$

Tabela 13 - Taxas de desconto 63

Tabela 14: Conversores de unidades e densidades 64

Tabela 15 - Valor Presente Líquido das plantas A e B sem

flexibilidade 65

Tabela 16 - Valor Presente Líquido da planta com flexibilidade de input 66

Tabela 17 - Perfis de produção por cenário $\quad 67$

Tabela 18 - Valor Presente Líquido dos diferentes perfis de produção 67

Tabela 19 - Valor Presente Líquido da planta com flexibilidade

de output 67

Tabela 20 - Valor Presente Líquido de uma planta Total Flex 68

Tabela 21 - Consolidação dos resultados 68

Tabela 22 - Tabela parametrizada ASF (Anderson-Schulz-Flory) 73

Tabela 23 - Resultados encontrados através da regressão linear $\quad 76$ 


\section{Lista de símbolos, abreviaturas e siglas}

GTL - gas to liquids

FT - Fischer- Tropsch

bbl - barris por dia

QP - Qatar Petroleum

p.p.m - partes por milhão

SMR - processo de reforma a vapor

POX - processo de oxidação parcial

HCC - Hidrocraking

HIDW -Hdrodewaxing

ANP - Agência Nacional do Petróleo

OPEC - Organization of the Petroleum Exporting Countries.

On Shore - dentro do continente

Off Shore - fora do continente, em alto mar

Input - entrada

Output - saída

Shut Down - processo de parada temporária

Restart - reinício de operação

Max[.] - (operador) máximo

Syngas - gás de síntese

$\pi-\mathrm{pi}, 3,14$

VPL - valor presente líquido

Prob[.] - (operador) probabilidade

$\varepsilon_{t}-$ variável aleatória

$\mathrm{dz}$ - incremento de Wiener

$\mathrm{dt}$ - variação de tempo infinitesimal

$\mathrm{dx}$ - variação de $\mathrm{x}$ infinitesimal

$\alpha-$ tendência (drift)

$\sigma-$ volatilidade

$\mathrm{E}[$.$] - (operador) valor esperado$

V[.] - (operador) variância

$M A B$ - movimento aritmético browniano

MGB - movimento geométrico browniano

MRM - movimento de reversão à média

MRMJ - movimento de reversão à média com saltos

$\eta$ - velocidade de reversão à média

$\bar{x}$ - média de longo prazo 
$\mathrm{e}^{[.]}-$(operador) exponencial

dq - distribição de probabilidade dos saltos aleatórios

$\lambda$ - taxa de ocorrência dos saltos aleatórios

$\varphi$ - tamanho dos saltos aleatórios

$\mathrm{Nm}^{3}$ - normal metro cúbico

ton MP - toneladas de matéria-prima

$\mathrm{I}_{0}-$ Investimento

Capex - capital expenditure

Opex - operational expenditure

CPV - custo dos produtos vendidos

ASF - curva Anderson-Schulz-Flory

Pis/Pasep - Programa de Integração social

Cofins - Contribuição para o Financiamento da Seguridade Social

ISS - Imposto Sobre Serviços

ICMS - Imposto sobre Circulação de Mercadorias e Serviços

IRPJ - Imposto de Renda sobre Pessoa Jurídica

CSLL - Contribuição Social sobre Lucro Líquido

MMBTU - milhões BTU (British Thermal Unit)

US\$c/gal - centavos de dólar por galão

I - litro

Ln - logaritmo na base e

$\mathrm{N}[$.$] - (operador) distribuição normal$

$\mu$ - taxa de desconto ajustada ao risco

$r_{f}-$ taxa de desconto livre de risco

p.a. - per annum, por ano

Alpha - perfil de produção ASF

Cenpes - Centro de Pesquisas e Desenvolvimento Leopoldo A. Miguez de Mello

@Risk - plataforma estatística

Out-of-the-money - opção fora do dinheiro 